\title{
Discovery of the short $\gamma$-ray burst GRB 050709
}

\author{
J. S. Villasenor ${ }^{1}$, D. Q. Lamb², G. R. Ricker ${ }^{1}$, J.-L. Atteia ${ }^{3}$, N. Kawai ${ }^{4}$, N. Butler ${ }^{1}$, Y. \\ Nakagawa $^{5}$, J. G. Jernigan ${ }^{6}$, M. Boer ${ }^{7}$, G. B. Crew ${ }^{1}$, T. Q. Donaghy ${ }^{2}$, J. Doty ${ }^{8}$,

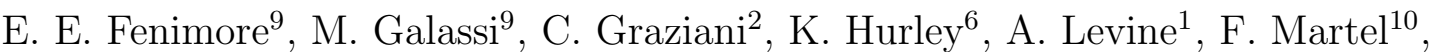 \\ M. Matsuoka ${ }^{11}$, J.-F. Olive ${ }^{7}$, G. Prigozhin ${ }^{1}$, T. Sakamoto ${ }^{12}$, Y. Shirasaki ${ }^{13}$, M. Suzuki ${ }^{14}$,

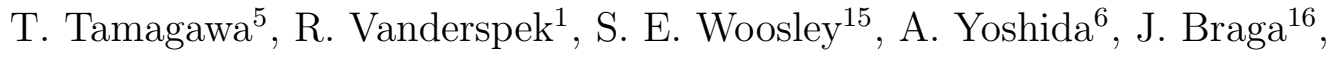 \\ R. Manchanda ${ }^{17}$, G. Pizzichini ${ }^{18}$, K. Takagishi ${ }^{19}$, and M. Yamauchi ${ }^{19}$,
}

Received _ ; accepted _ 
${ }^{1}$ MIT Kavli Institute, Massachusetts Institute of Technology, 70 Vassar Street, Cambridge, MA, 02139, USA.

${ }^{2}$ Department of Astronomy and Astrophysics, University of Chicago,5640 South Ellis Avenue, Chicago, IL 60637, USA.

${ }^{3}$ Laboratoire d'Astrophysique, Observatoire Midi-Pyrénées, 14 Ave. E. Belin, 31400 Toulouse, France.

${ }^{4}$ Department of Physics, Tokyo Institute of Technology, 2-12-1 Ookayama, Meguro-ku, Tokyo 152-8551, Japan.

${ }^{5}$ Department of Physics and Mathematics, Aoyama Gakuin University, Fuchinobe 5-10-1, Sagamihara, Kanagawa 229-8558, Japan.

${ }^{6}$ University of California at Berkeley, Space Sciences Laboratory, Berkeley, CA, 94720-7450, USA.

${ }^{7}$ Centre d'Etude Spatiale des Rayonnements, Observatoire Midi-Pyrénées, 9 Ave. de Colonel Roche, 31028 Toulouse Cedex 4, France.

${ }^{8}$ Noqsi Aerospace, Ltd., 2822 South Nova Road, Pine, CO 80470, USA.

${ }^{9}$ Los Alamos National Laboratory, P.O. Box 1663, Los Alamos, NM, 87545, USA.

${ }^{10}$ Espace Inc., 30 Lynn Avenue, Hull, MA 02045, USA.

${ }^{11}$ Tsukuba Space Center, Japan Aerospace Exploration Agency, Tsukuba, Ibaraki, 305-8505, Japan.

${ }^{12}$ NASA Goddard Space Flight Center, Greenbelt, MD, 20771, USA.

${ }^{13}$ National Astronomical Observatory, Osawa 2-21-1, Mitaka, Tokyo 181-8588 Japan.

${ }^{14}$ RIKEN (Institute of Physical and Chemical Research), 2-1 Hirosawa, Wako, Saitama 351-0198, Japan. 
${ }^{15}$ Department of Astronomy and Astrophysics, University of California at Santa Cruz, 477 Clark Kerr Hall, Santa Cruz, CA 95064, USA.

${ }^{16}$ Instituto Nacional de Pesquisas Espaciais, Avenida Dos Astronautas 1758, São José dos Campos 12227-010, Brazil.

${ }^{17}$ Department of Astronomy and Astrophysics, Tata Institute of Fundamental Research, Homi Bhabha Road, Mumbai, 400 005, India.

${ }^{18}$ INAF/IASF Bologna, Via Gobetti 101, 40129 Bologna, Italy.

${ }^{19}$ Faculty of Engineering, Miyazaki University, Gakuen Kibanadai Nishi, Miyazaki 889-2192, Japan. 
Gamma-Ray Bursts (GRBs) fall into two classes: short-hard and long-soft bursts $^{1,2,3}$. The latter are now known to have $\mathrm{X}-\mathrm{ray}^{4}$ and optical afterglows ${ }^{5}$, to occur at cosmological distances ${ }^{6}$ in star-forming galaxies ${ }^{7}$, and to be associated with the explosion of massive $\operatorname{stars}^{8,9}$. In contrast, the distance scale, the energy scale, and the progenitors of short bursts have remained a mystery. Here we report the discovery of a short-hard burst whose accurate localization has led to follow-up observations that have identified the X-ray afterglow ${ }^{10}$ and (for the first time) the optical afterglow ${ }^{10-11}$ of a short-hard burst. These, in turn, have led to identification of the host galaxy of the burst as a late-type galaxy at $z=0.16,{ }^{10}$ showing that at least some short-hard bursts occur at cosmological distances in the outskirts of galaxies, and are likely to be due to the merging of compact binaries.

On 9 July 2005, at 22:36:37 UT, the Soft X-Ray Camera (SXC), Wide-Field X-Ray Monitor (WXM) and French Gamma Telescope (FREGATE) instruments on board the High Energy Transient Explorer 2 satellite (hereafter HETE ${ }^{12}$ ) detected GRB 050709, a short-hard pulse followed by a long-soft bump from the same location. Figure 1 shows the WXM and SXC localizations for the burst, and the location of the X-ray and optical afterglows. Figures 2 and 3 show the time history of the entire burst and of the short-hard pulse in several energy bands.

Figure 4 compares the best-fit spectral model and the spectral data for the short-hard pulse. Table 1 gives the best-fit spectral parameters for different time intervals during the burst. The spectrum of the short pulse is hard and that of the intense first peak of the pulse (corresponding to the first $0.2 \mathrm{~s}$ of the burst) is even harder.

The duration and the peak energy $E_{\text {peak }}^{\text {obs }}$ of the spectrum of the short-hard pulse are consistent with those of short-hard GRBs. ${ }^{13,14}$ We note that its duration is much shorter 
and its spectrum is much harder than these were for GRB 020531, the other short-hard burst localized by HETE. ${ }^{15}$ We also note that the time history and the spectral properties of GRB 050709 are similar to those of several Burst and Transient Source Experiment (BATSE) bursts, including GRBs 921022, 990516, and 990712 ${ }^{16,17}$ (see Table 1). Table 2 gives the emission properties of the burst. The gamma-ray to X-ray fluence ratio of the short-hard pulse is 3.1, which is also consistent with those of BATSE short-hard bursts.

The isotropic-equivalent energy $E_{\text {iso }}$ of the short-hard pulse is a factor $\sim 1000$ smaller than is typical of long GRBs and implies that the energy $E_{\gamma}$ radiated by the short-hard pulse in $\gamma$ rays is at least 40 times less than is typical for long GRBs. ${ }^{18}$ The very small value of $E_{\text {iso }}$ also places the short-hard pulse off of the $E_{\text {iso }}-E_{\text {peak }}$ relation found by Refs. 19 and 20 for long GRBs by a factor of $\sim 1000$ in $E_{\text {iso }}$. The luminosity $L_{\text {iso }}$ of the short-hard pulse is also very small, and places it off of the $L_{\text {iso }}-E_{\text {peak }}$ correlation found by Refs. 20 and 21 for long GRBs by a factor of $\sim 100$ in $L_{\text {iso }}$. These three results strengthen the conclusion that GRB 050709 is not a long-soft burst.

There is evidence that many short-hard bursts observed by the BATSE and Konus instruments exhibit a long-soft bump following the initial hard pulse that is similar to that seen by HETE in GRB 050709. ${ }^{22-24}$ We have already mentioned the similarity of the time history and spectral properties of GRB 050709 and those of GRBs 921022, 990516, and 990712. Refs. 22 and 23 reported evidence that BATSE and Konus short-hard bursts, respectively, are followed by a 30-200 s period of long-soft emission having a spectrum that is consistent with that of the long-soft bump in GRB 050709, while Ref. 24 reported evidence that BATSE short-hard bursts show an excess of soft emission from 20 s to 100-300 s after the burst.

The fluence of the long-soft bump is much greater than that of the short-hard pulse, unlike what is seen in SGR giant flares. ${ }^{25,26}$ In addition, we have analyzed the time 
history of the long-soft bump and find no evidence for brightness oscillations of the kind that characterize the long-soft bump of SGR giant flares ${ }^{25,26}$ (see Figure 2); however, we can place only a weak constraint on the amplitude of any such oscillations because the signal-to-noise ratio of the light curve of the long-soft bump is low. We have also searched for any evidence of stochastic variability of the long-soft bump and find none (also see Figure 2). Finally, we have searched for evidence of spectral evolution during the long-soft bump and find none (see Table 1).

The most natural interpretation of the long-soft bump is that it is the beginning of the afterglow. Its time history and spectrum are consistent with those expected for an afterglow, as is the lack of any time variability or spectral evolution. The ratio of the fluence in the short-hard pulse to that in the short-hard pulse plus the long-soft bump implies a radiative efficiency of $<25 \%$ for the prompt phase. If the peak of the long-soft bump at $\approx 100 \mathrm{~s}$ corresponds to the time at which the fireball decelerates, a consistent solution exists in which $z=0.16$, the GRB jet has an isotropic-equivalent kinetic energy $E_{\mathrm{KE}} \sim 5 \times 10^{49}$ erg, a relativistic bulk $\Gamma \approx 100$, and expands into a low-density medium having a number density $n \sim 10^{-2} \mathrm{~cm}^{-3}$ (see Figure 2).

The accurate location of GRB $050509 \mathrm{~b}$ by the BAT and XRT on board Swift led for the first time to the identification of the X-ray afterglow of a short GRB. ${ }^{27}$ However, the burst occurred in the direction of two merging clusters of galaxies, making it impossible to determine the host galaxy of the burst and thus the redshift of the burst ( $>20$ galaxies lie within the XRT error circle for the X-ray afterglow of the burst), let alone the location of the burst within the host galaxy.

The accurate location of GRB 050709 by the WXM and SXC on board HETE has led to the identification of the X-ray afterglow, ${ }^{10}$ and for the first time, the identification in ground-based $^{10-11}$ and HST ${ }^{11}$ images of the optical afterglow of a short-hard burst. These 
have led to the first secure identification of a host galaxy: a late-type spiral galaxy lying at a redshift $z=0.16 .{ }^{11}$ The X-ray and optical afterglows lie at a projected distance of $\approx 3$ kpc from the center of the host galaxy and are therefore not coincident with the brightest optical emission from the host galaxy (in contrast to long bursts ${ }^{28}$ ).

These results constrain the nature of the central engine for GRB 050709, and by implication all short-hard bursts. The absence of any large-amplitude oscillations with a period in the range 1-10 s in the long-soft bump and the offset of GRB 050709 from the center of the host galaxy argue against an association between this short-hard burst and SGR giant flares. ${ }^{25,26}$ Models based upon core collapse in massive stars ${ }^{9}$ explain the association of some long-soft bursts with supernovae. However, given the time it takes for the GRB-producing jet to emerge from a collapsing massive star, it is difficult for such models to produce bursts shorter than a few seconds. Merging neutron stars, on the other hand, can produce very short bursts. ${ }^{29}$ Given the smaller mass of the accretion disk that forms, it is not unreasonable to expect a lower average $E_{\text {iso }}$ for short-hard bursts, and hence a smaller average redshift. Moreover, since binary neutron stars are imparted with a "kick" at birth and travel large distances before merging, one expects an offset of order several kpc between the star-forming regions of the host galaxy and the burst. ${ }^{30}$ GRB 050709 exhibits all of these properties. The roughly $100 \mathrm{~s}$ lag between the short-hard pulse of GRB 050709 and the peak of the much softer afterglow is consistent with the low-density interstellar medium expected in the vicinity of a merging compact binary. ${ }^{22}$ If short-hard GRBs are due to merging neutron stars, they produce powerful bursts of gravitational radiation that should be detectable by the second-generation Laser Interferometry Gravitational-Wave Observatory.

The HETE localization of the short-hard burst GRB 050709 has led to follow-up observations that have identified the X-ray afterglow and (for the first time) the optical 
afterglow of a short, hard burst. These, in turn, have led to identification of the host galaxy of the burst as a late-type galaxy at $z=0.16$, showing that at least some short-hard bursts occur at cosmological distances in the outskirts of galaxies, and are likely to be due to the merging of compact binaries. 
1. Hurley, K. in Gamma-Ray Bursts (ed. W. Paciesas \& G. Fishman) 3 (AIP, New York, 1992)

2. Lamb, D. Q., Graziani, C. \& Smith, I. Evidence for two distinct morphological classes of gamma-ray bursts from their short time scale variability. Astrophys. J. 413, L11-14 (1993)

3. Kouveliotou, C., et al. Identification of two classes of gamma-ray bursts. Astrophys. J. 413, L101-104 (1993)

4. Costa, E., et al. Discovery of an X-ray afterglow associated with the gamma-ray burst of 28 February 1997. Nature 387, 783-784 (1997)

5. van Paradijs, J. et al. Transient optical emission from the error box of the gamma-ray burst of 28 February 1997. Nature 386, 686-688 (1997)

6. Metzger, M R. et al. Spectral constraints on the redshift of the optical counterpart to the gamma-ray burst of 8 May 1997. Nature 387, 878-879 (1997)

7. Castander, F. \& Lamb, D. Q. A Photometric Investigation of the GRB 970228 Afterglow and the Associated Nebulosity. Astrophys. J. 523, 593-601 (1999)

8. Woosley, S. E. Gamma-ray bursts from stellar mass accretion disks around black holes. Astrophys. J. 405, 273l-277 (1993)

9. Stanek, C., et al. Spectroscopic Discovery of the Supernova 2003dh Associated with GRB 030329. Astrophys. J. 591, L17-20 (2003)

10. Fox, D. B., et al. ... Nature, submitted

11. Hjorth, J., et al. The optical afterglow of a short $\gamma$-ray burst. Nature, submitted

12. Ricker, G. R., et al. in Gamma-Ray Burst and Afterglow Astronomy 2001: A 
Workshop Celebrating the First Year of the HETE Mission (ed. G. R. Ricker and R. K. Vanderspek) 3-16 (AIP Press, New York, 2003)

13. Paciesas, W., et al. in Gamma-Ray Bursts in the Afterglow Era (ed. E. Costa, F. Frontera and J. Hjorth) 13-15 (Springer, Berlin, 2000)

14. Ghirlanda, G., Ghisellini, G. \& Celotti, A., The spectra of short gamma-ray bursts. Astron. Astrophys. 422, L55-58 (2004)

15. Lamb, D. Q., et al. in Gamma-Ray Bursts in the Afterglow Era (ed. M. Feroci, F. Frontera, N. Masetti, and L. Piro) 94-97 (ASP, San Francisco, 2004).

16. Paciesas, W., et al. The Fourth BATSE Gamma-Ray Burst Catalog (Revised). Astrophys. J. Supp. 122, 465-495 (2000)

17. Preece, R., et al. The BATSE Gamma-Ray Burst Spectral Catalog. I. High Time Resolution Spectroscopy of Bright Bursts Using High Energy Resolution Data. Astrophys. J. Supp. , 126, 19-36 (2000)

18. Frail, D. A., et al. Beaming in Gamma-Ray Bursts: Evidence for a Standard Energy Reservoir. Astrophys. J. 562, L55-58 (2001)

19. Amati, L., et al. Intrinsic spectra and energetics of BeppoSAX Gamma-Ray Bursts with known redshifts. Astron. Astrophys. 390, 81-89 (2002)

20. Lamb, D. Q., et al. Scientific Highlights of the HETE-2 Mission. New Astron. Rev. 48, 423-430 (2004)

21. Yonetoku, D., et al. Gamma-Ray Burst Formation Rate Inferred from the Spectral Peak Energy-Peak Luminosity Relation. Astrophys. J. 609, 935-951 (2004)

22. Lazzati, D., Ramirez-Ruiz, E. \& Ghisellini, G. Possible detection of hard X-ray afterglows of short $\gamma$-ray bursts. Astron. Astrophys. 379, L39-43 (2001) 
23. Frederiks, D. D., et al. in Gamma-Ray Bursts in the Afterglow Era (ed. M. Feroci, F. Frontera, N. Masetti, and L. Piro) 197-200 (ASP, San Francisco, 2004).

24. Connaughton, V. BATSE Observations of Gamma-Ray Burst Tails. Astrophys. J. 567, 1028-1036 (2002)

25. Hurley, K., et al. An exceptionally bright flare from SGR 1806-20 and the origin of short duration gamma-ray bursts. Nature 434, 1098-1103 (2005)

26. Palmer, D. M., et al. A giant gamma-ray flare from the magnetar SGR 1806-20. Nature 434, 1107-1109 (2005)

27. Gehrels, N., et al. The first short gamma-ray burst localized by Swift. Nature , (2005)

28. Bloom, J., et al. The Observed Offset Distribution of Gamma-Ray Bursts from Their Host Galaxies: A Robust Clue to the Nature of the Progenitors. Astron. J. 123, 1111-1148 (2002)

29. Eicher, D., Livio, M., Piran, T., \& Schramm, D. N. Nucleosynthesis, neutrino bursts and $\gamma$-rays from coalescing neutron stars. Nature 340, 126-128 (1989)

30. Fryer, C. L., Woosley, S. E. \& Hartmann, D. H. Formation Rates of Black Hole Accretion Disk Gamma-Ray Bursts. Astrophys. J. , 526, 152-177 (1999)

31. Reichart, D. E., et al. A Possible Cepheid-like Luminosity Estimator for the Long Gamma-Ray Bursts. Astrophys. J. 552, 57 (2001)

Acknowledgements This research was supported in the U.S. by NASA.

Author Information The authors declare no competing financial interests. Correspondence should be addressed to G.R.R. (grr@space.mit.edu) or to J.S.V. (jsvilla@space.mit.edu). 
Table 1. Spectral Model Parameters for GRB 050709.

\begin{tabular}{lcccc}
\hline \hline \multicolumn{1}{c}{ Parameter } & $\mathrm{t}=0-0.20 \mathrm{~s}$ & $\mathrm{t}=0.20-0.50 \mathrm{~s}$ & $\mathrm{t}=0-0.50 \mathrm{~s}$ & $\mathrm{t}=20-180 \mathrm{~s}$ \\
\hline & & & & \\
Spectral Model: & PLE & PLE & PLE & PL \\
Photon index $\alpha$ & $0.53_{-0.13}^{+0.12}$ & $0.55_{-1.3}^{+1.0}$ & $0.82_{-0.14}^{+0.13}$ & $1.98_{-0.15}^{+0.18}$ \\
Peak energy (E $\left.E_{\text {peak }}^{\text {obs }}\right)$ & $83.9_{-8.3}^{+11}$ & $10.6_{-3.5}^{+4.5}$ & $86.5_{-11}^{+16}$ & - \\
Normalization (at 15 keV) & $0.79_{-0.08}^{+0.07}$ & $0.650_{-0.48}^{+3.24}$ & $0.377_{-0.04}^{+0.04}$ & $0.0075_{-0.0013}^{+0.0013}$ \\
Chi-squared (DOF) & $439(366)$ & $374(366)$ & $467(366)$ & $336(367)$ \\
\hline
\end{tabular}

Note. - The spectral models are power-law times exponential (PLE) and power-law (PL). Errors are for $90 \%$ confidence. The normalization units are photons $\mathrm{cm}^{-2} \mathrm{~s}^{-1} \mathrm{keV}^{-1}$. DOF is the number of degrees of freedom in the fit. We have also fit PL and Band models to the first three time intervals and find $\chi^{2}$ (DOF) values of 550 (367), 383 (367), and 538 (367) for the PL model and 439 (365), 374 (365), and 467 (365) for the Band model, demonstrating that the data request the PLE model but not the Band model. Similarly, we have also fit a PLE model to the fourth time interval and find $\chi^{2}$ (DOF) values of 336 (366), demonstrating that the data request the PL model but not the PLE model. The large values of $\chi^{2}$ per DOF that we find for the PLE model for the 0-0.2 s and 0-0.5 s time intervals are due to the rapid spectral evolution during the short-hard pulse. We have calculated the hardness ratio $H=$ counts(5-10 keV)/counts(2-5 keV) for 10, 20,30, and $40 \mathrm{~s}$ time intervals and find no evidence of spectral evolution during the long-soft bump. The time history and spectral parameters of GRB 050709 are similar to those of the BATSE bursts GRBs 921022, 990516, and 990712..17,18 
In particular, the short-hard pulse of GRB 921022 had a duration $\approx 256 \mathrm{~ms}$ and spectral parameters $\alpha=-1.1 \pm 0.3, \beta=-2.15 \pm 0.12$, and $E_{\text {peak }}^{\text {obs }}=123 \pm 28 \mathrm{keV}$, and the long-soft bump of that burst had a PL spectrum with index $\alpha \approx-2 ;{ }^{17}$ while the short-hard pulse of GRB 990712 had a duration $\approx 0.75 \mathrm{~s}$ and spectral parameters $\alpha \approx-0.2$ and $E_{0} \approx 600 \mathrm{keV}$, and the long-soft bump of that burst had a PL spectrum with index $\alpha=-1.9 \pm 0.6 .^{23}$ 
Table 2. Emission Properties of GRB 050709.

\begin{tabular}{|c|c|c|c|c|}
\hline $\begin{array}{l}\text { Energy } \\
(\mathrm{keV})\end{array}$ & $\begin{array}{l}\text { Peak Photon Flux } \\
\left(\mathrm{ph} \mathrm{cm}^{-2} \mathrm{~s}^{-1}\right)\end{array}$ & $\begin{array}{l}\text { Photon Fluence } \\
\qquad\left(\mathrm{ph} \mathrm{cm}^{-2}\right)\end{array}$ & $\begin{array}{l}\text { Peak Energy Flux } \\
\left(10^{-8} \mathrm{erg} \mathrm{cm}^{-2} \mathrm{~s}^{-1}\right)\end{array}$ & $\begin{array}{l}\text { Energy Fluence } \\
\left(10^{-8} \mathrm{erg} \mathrm{cm}^{-2}\right)\end{array}$ \\
\hline \multicolumn{5}{|l|}{ Short pulse: } \\
\hline $2-10$ & $29.0 \pm 5.2$ & $3.47 \pm 0.59$ & $24.9 \pm 3.9$ & $2.81 \pm 0.42$ \\
\hline $2-25$ & $53.6 \pm 6.1$ & $5.55 \pm 0.69$ & $88.7 \pm 7.7$ & $8.31 \pm 0.70$ \\
\hline $2-30$ & $58.1 \pm 6.9$ & $5.94 \pm 0.70$ & $111 \pm 8.6$ & $10.1 \pm 0.76$ \\
\hline $7-30$ & $37.1 \pm 2.8$ & $3.31 \pm 0.25$ & $96.8 \pm 6.8$ & $8.35 \pm 0.59$ \\
\hline $30-400$ & $34.1 \pm 2.7$ & $2.51 \pm 0.22$ & $400 \pm 46$ & $30.3 \pm 3.8$ \\
\hline $50-100$ & $13.9 \pm 1.1$ & $0.986 \pm 0.087$ & $156 \pm 13$ & $11.0 \pm 1.0$ \\
\hline $100-300$ & $6.62 \pm 1.1$ & $0.515 \pm 0.092$ & $155 \pm 29$ & $12.4 \pm 2.5$ \\
\hline $2-400$ & $92.1 \pm 7.6$ & $8.43 \pm 0.752$ & $511 \pm 49$ & $40.3 \pm 4.1$ \\
\hline \multicolumn{5}{|l|}{ Long bump: } \\
\hline $2-10$ & $2.36 \pm 0.43$ & $107 \pm 18$ & $1.53 \pm 0.27$ & $69.1 \pm 10$ \\
\hline $2-25$ & $2.72 \pm 0.47$ & $123 \pm 18$ & $2.41 \pm 0.37$ & $109 \pm 14$ \\
\hline
\end{tabular}

Note. - The quantities in this table are derived assuming the best-fit PLE model for the spectrum of the short-hard pulse and the best-fit PL model for the spectrum of the long-soft bump. Errors are $90 \%$ confidence level. The photon number and photon energy peak fluxes for the short-hard pulse were evaluated over a $70 \mathrm{~ms}$ interval, corresponding to $T_{90}$ for the short-hard pulse; those for the long-soft bump are evaluated in a $1 \mathrm{~s}$ interval. 
Using the redshift $z=0.16$ measured for the host galaxy, ${ }^{11}$ the isotropic-equivalent energy of the short-hard pulse in the $1-10,000 \mathrm{keV}$ energy band in the rest frame of the source is $E_{\text {iso }}=\left(2.8_{-0.2}^{+0.4}\right) \times 10^{49} \mathrm{erg}$, taking $\Omega_{M}=0.3, \Omega_{\Lambda}=0.7$, and $h=0.65$. Using a time interval $0.060 \mathrm{~s}$ in the rest frame of the source (corresponding to a duration of $0.07 \mathrm{~s}$ in the observer frame) and assuming the same cosmology and energy band, the luminosity of the short-hard pulse in the $1-10,000 \mathrm{keV}$ energy band in the rest frame of the source is $L_{\text {iso }}=(5.2 \pm 0.7) \times 10^{50} \mathrm{erg} \mathrm{s}^{-1}$. 


\section{FIGURE CAPTIONS}

Fig. 1.-Sky map showing the HETE localization error circles for GRB 050709 and the location of the X-ray and optical afterglow. The WXM obtained a localization in flight. However, the spacecraft attitude-control system was not locked at the time of the trigger, resulting in a drift of the satellite pointing direction, and real-time aspect was not available. Consequently, the location was not distributed in real time. Ground analysis of the data from the optical cameras provided reliable spacecraft aspect information, despite the spacecraft drift rate. A GCN Notice was sent out at 22:00:09 UT on 10 July 2005, after ground determination of the spacecraft aspect. Ground analysis of the WXM data produced a location with a $90 \%$ confidence region that is a circle centered at R.A. $=+23 \mathrm{~h} 01 \mathrm{~m} \mathrm{44s}$; Dec. $=-38^{\circ} 59^{\prime} 52^{\prime \prime}(\mathrm{J} 2000)$ with a radius of $14.5^{\prime}$ (large circle labelled "WXM-ground"). Ground analysis of the SXC data yielded a refined location with a $90 \%$ confidence region that is a circle centered at R.A. $=+23 \mathrm{~h} 01 \mathrm{~m} \mathrm{30s} ;$ Dec. $=-38^{\circ} 58^{\prime}$

$33^{\prime \prime}$ (J2000) with a radius of $1.34^{\prime}$ (small circle labelled "SXC-ground"). The location of the X-ray and optical afterglow is labelled "afterglow."

Fig. 2.-Time history of GRB 050709. From top to bottom: Time history observed by WXM in the 2-10 keV energy band (a) and in the 2-25 keV energy band (b); time history observed by FREGATE in the 6-40 keV energy band (c) and in the 30-400 keV energy band (d). The event is a short-hard spike of duration $T_{90}=220 \pm 50 \mathrm{~ms}$ in the $2-25 \mathrm{keV}$ energy band and $70 \pm 10 \mathrm{~ms}$ in the 30-400 keV energy band, followed $\sim 25$ seconds later by a long-soft bump of duration $T_{90}=130 \pm 7 \mathrm{~s}$ in the $2-25 \mathrm{keV}$ energy band - where $T_{90}$ is the time interval containing $90 \%$ of the photons. We have performed an FFT on the time history of the long-soft bump for the time interval 5-175 s after the trigger. We find no evidence for any coherent brightness oscillations in the period range 1-10 s, and derive $3 \sigma$ upper limits on the amplitude of any such oscillations of $77 \%$ and $89 \%$ at periods of 1 and 5 s, respectively. 
We have also computed the variability measure $V,{ }^{27}$ for the long-soft bump, using smoothing time scales of 10, 20, 30, and 40s. We find $V=0.005 \pm 0.013,0.003 \pm 0.026,0.007 \pm 0.031$, and $0.025 \pm 0.036$, respectively. We therefore find no evidence for a non-zero value of $V$. If the long-soft bump is the afterglow and its peak at $t_{\text {peak }} \approx 100 \mathrm{~s}$ corresponds to the time at which the fireball decelerates, a consistent solution exists in which $z=0.16$; the GRB jet has an isotropic-equivalent kinetic energy $E_{\mathrm{KE}}=\left(1-\cos \theta_{\text {jet }}\right) E_{\text {iso }}{ }^{\text {total }} / \eta \sim 5 \times 10^{49}$ erg

- where $E_{\text {iso }}{ }^{\text {total }}=E_{\text {iso }}($ pulse $)+E_{\text {iso }}($ bump $) \sim 1 \times 10^{50} \mathrm{erg}, \theta_{\text {jet }} \sim 0.3$ is the jet opening angle, ${ }^{18}$ and $\eta=0.2$ is the radiative efficiency; a relativistic bulk $\Gamma \approx 100$; and expands into a low-density medium having a number density $n \sim 10^{-2} \mathrm{~cm}^{-3} \cdot{ }^{22}$

Fig. 3.-Time history of the short-hard pulse of GRB 050709. From top to bottom: Time histories observed by the WXM in 2-10 kev energy band (a) and 10-25 keV band (b); and by FREGATE in 6-30 keV energy band (c), 30-85 keV energy band (d), and 85-400 $\mathrm{keV}$ energy band (e), plotted in $5 \mathrm{~ms}$ time bins. The pulse has a duration $T_{90}=220 \pm$ $50 \mathrm{~ms}$ in the $2-25 \mathrm{keV}$ energy band and $70 \pm 10 \mathrm{~ms}$ in the $30-400 \mathrm{keV}$ energy band, and exhibits no detectable emission before $T=0$ or after $T=400 \mathrm{~ms}$, confirming the short, hard nature of the pulse.

Fig. 4.-Comparison of the observed count spectrum and the best-fit PLE model for the short-hard pulse of GRB 050709. Upper panel: comparison of the counts in the WXM energy loss channels (lower energies) and the FREGATE energy loss channels (higher energies) and those predicted by the best-fit PLE model (smooth curves). Error bars are one sigma (i.e. $68 \%$ confidence limits). Lower panel: residuals to the fit. Error bars are one sigma. The short-hard pulse exhibits emission at all energies, confirming that its spectrum is hard. 


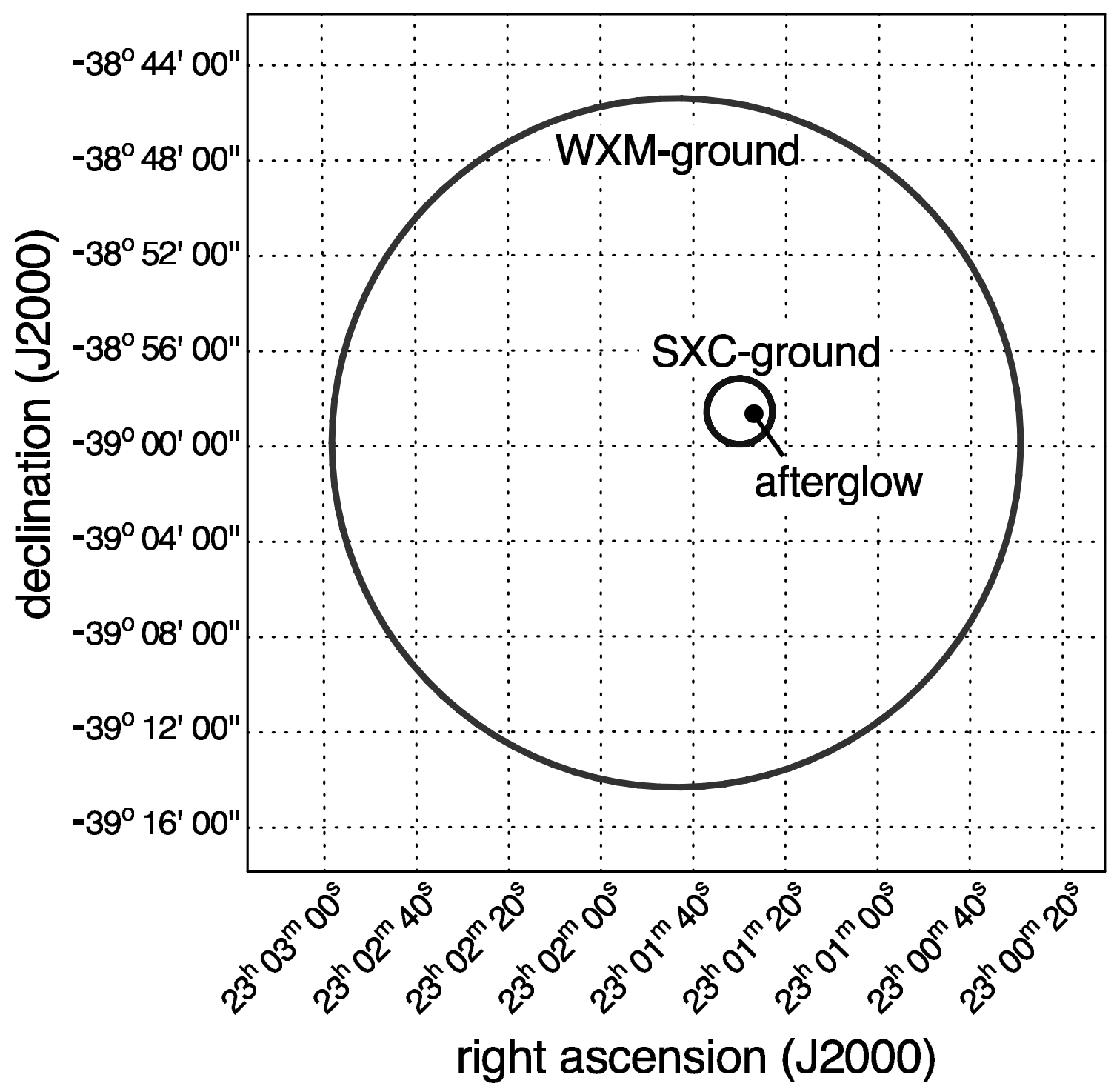




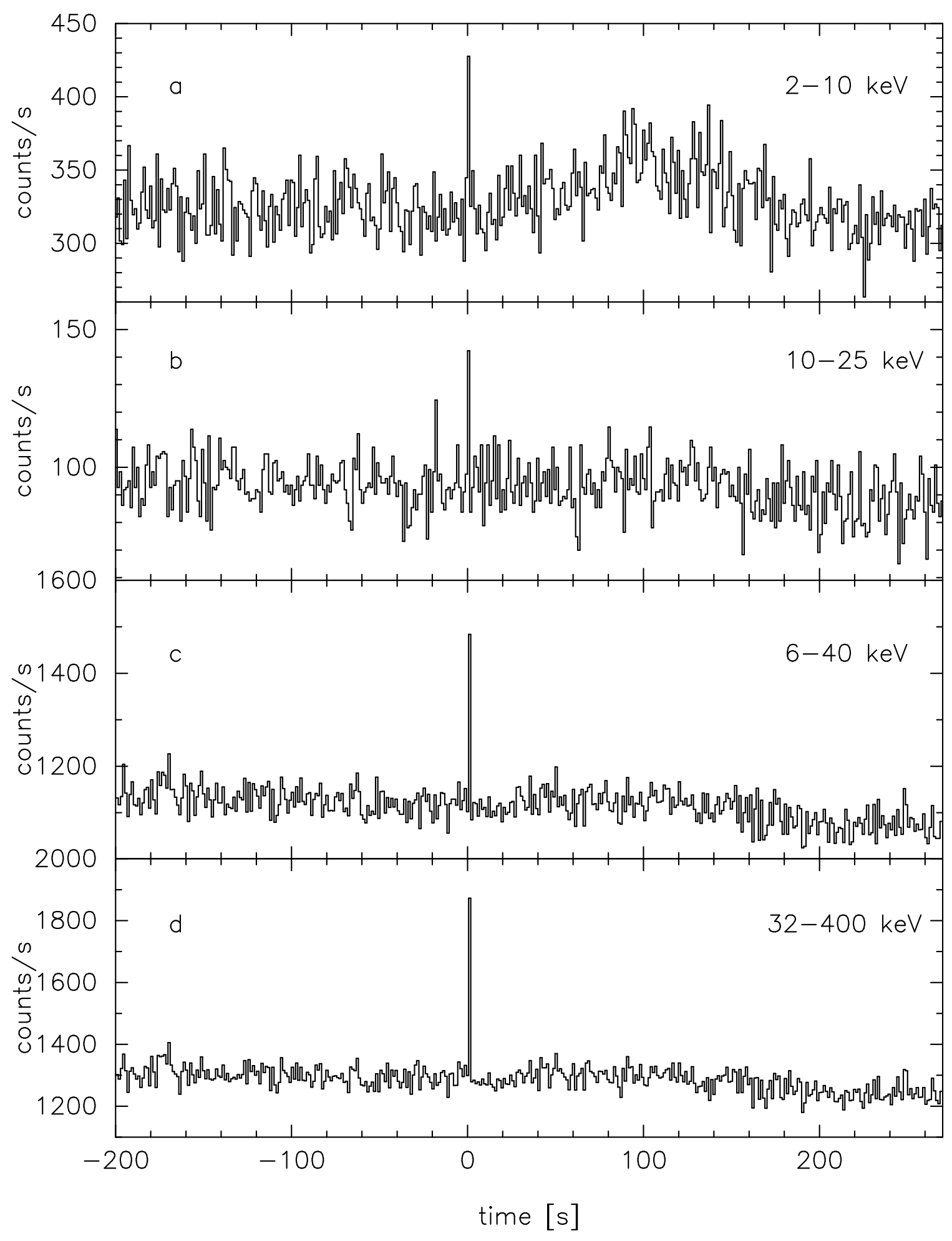




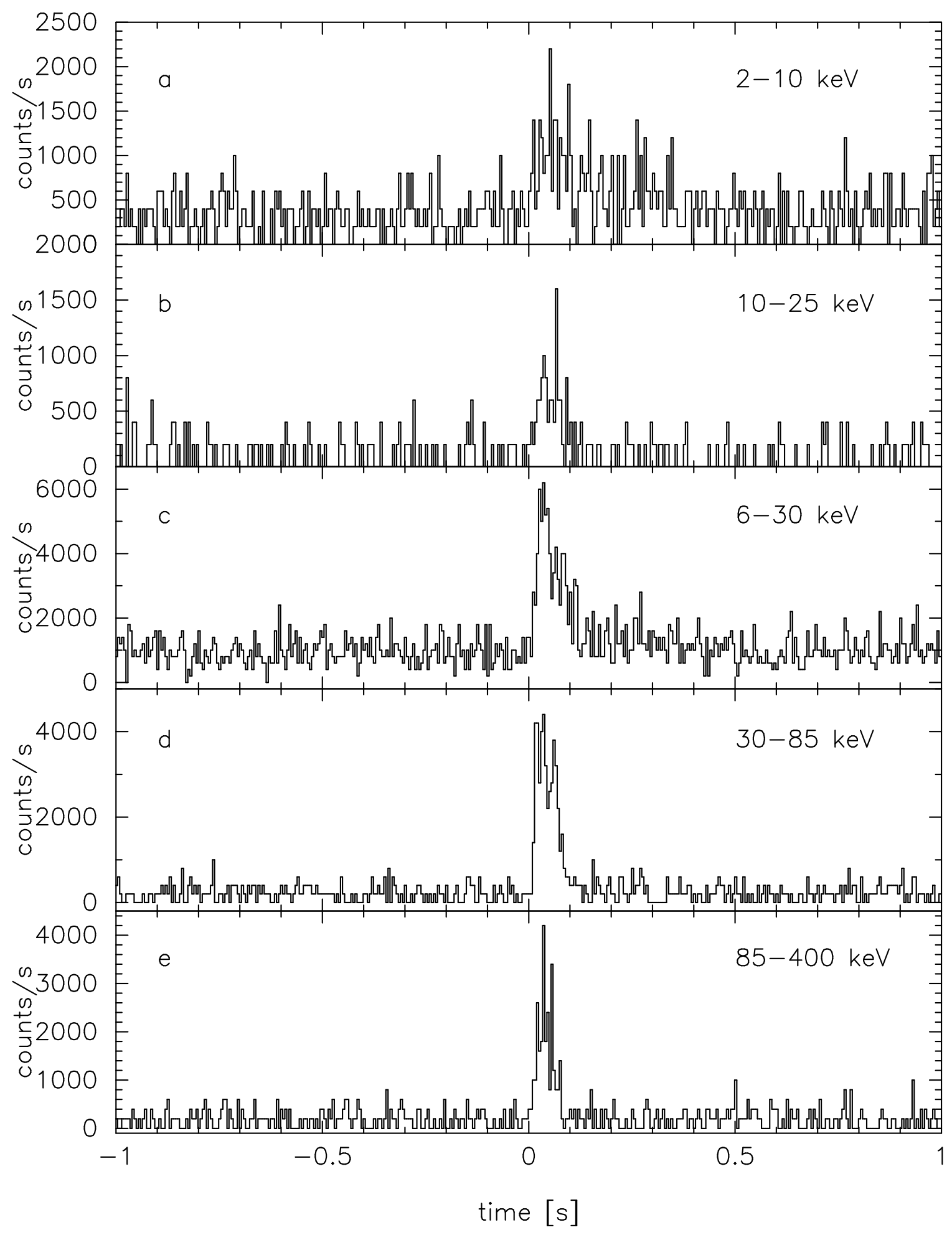




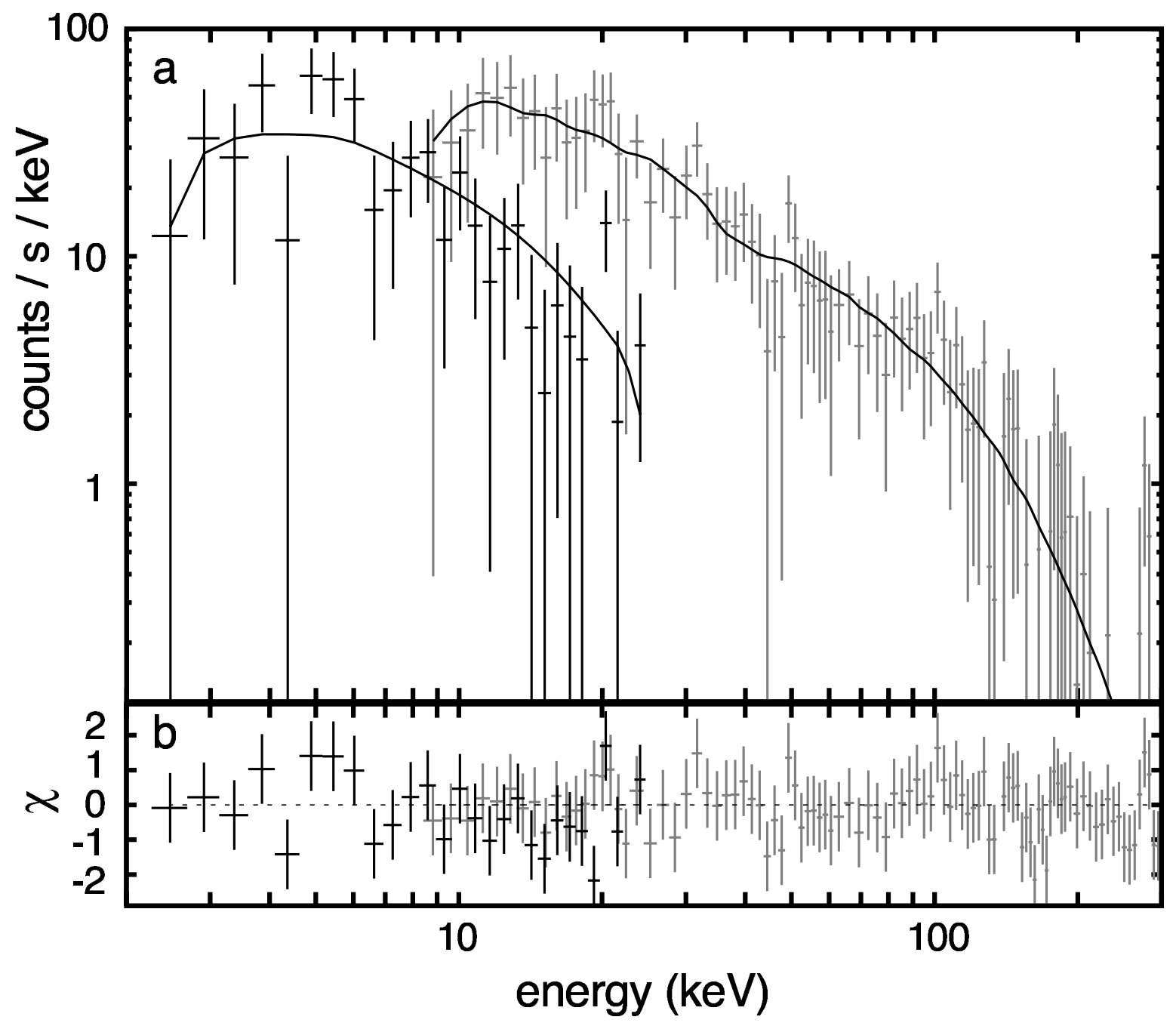

